\title{
Editorial
}

\section{Clubs, Carrots, and Conservation}

\section{Bâtons, carottes et conservation}

\author{
$\underline{\text { Marc-André Villard }}^{1}$ and $\underline{\text { Thomas D. Nudds }}^{2}$
}

Key Words: adaptive management; Environment Canada; incentive-based conservation; incidental take; Migratory Birds Convention Act

Despite increasing awareness of the impact of humans on ecosystems, many organisms are killed incidentally by activities that may potentially affect vital rates of populations. With regard to songbirds, examples include nest destruction by agricultural practices and timber harvest operations during summer. Just as commercial fishing bycatch may significantly alter oceanic food webs (Myers and Worm 2005), "incidental take" of birds by these or other activities, such as collisions with vertical structures or predation by domestic animals, might have significant cumulative effects on certain species or ecosystems. While the apparent effects of such activities typically loom larger in the public mind than habitat destruction or pesticide use during the nonbreeding season, scientific assessments are nevertheless required to determine whether, why, and to what extent their effects actually are harmful.

Environment Canada (EC) undertook exactly these types of assessments following an investigation by the Commission for Environmental Cooperation (created by the North American Free Trade Act) for an alleged failure by the Canadian government to enforce the Migratory Birds Convention Act (MBCA). The MBCA prohibits taking migratory birds, their nests, or their eggs by any means and at any time. However, in October 2010, a letter from senior management of EC's Environmental Stewardship Branch informed "stakeholders and interested parties" that, after three years of scientific research into the effects of the myriad ways that birds and their nests can be taken incidentally, EC would "not be requesting regulatory amendments,

\footnotetext{
${ }^{1}$ Université de Moncton, Canada, ${ }^{2}$ University of Guelph
}

but will focus on the development of practices that provide tangible support to compliance with the existing prohibitions. In addition, we will continue to work with interested parties to ensure a set of best practices that will help sustain populations of migratory birds in Canada and provide a solid basis for those that will want to use them."

From this information alone, it would be prejudicial to conclude anything about the fate of the scientific research into these matters that has been conducted by EC staff. Perhaps it was deemed too onerous to try to regulate incidental take in all its manifest forms, given available resources in government. Perhaps there were even bi-national considerations, which is frequent where the Migratory Birds Convention Act is concerned. Had EC issued a more transparent letter, stakeholders and interested parties on the outside would not be left to surmise the motives for the decision. Regardless, it is apparent that a regulatory approach will not be followed to address incidental take. Instead, EC will entertain propositions on best management practices from interested parties on a voluntary basis.

What is not clear is whether the apparent move from "clubs" is really to "carrots" (as an optimist might see it), i.e., using incentives to promote conservation action. Nor is it clear what role scientific research might play in assessing the efficacy of incentivebased conservation initiatives. Even laws like the MBCA, that offer relatively "expansive" language (Adkins Giese 2010) to protect migratory birds, still

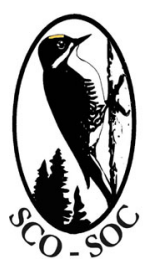

Sponsored by the Society of Canadian Ornithologists and Bird Studies Canada Parrainée par la Société des onithologistes du Canada et Études d'oiseaux Canada

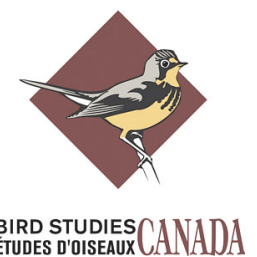


authorize government to allow the taking of migratory birds that is compatible with the terms of the legislation and carries out the purposes of the MBCA, for example as part of scientific research, as long as it is approved by authorized bodies. So does endangered species legislation in Canada (and at least one of its provinces, Ontario) allow government to permit activities, as long as the longterm effects of such activities are predicted to benefit species. We hope that the decision not to request regulatory amendments to the MBCA at least signals the government's intent to permit exceptions that will assist scientific analysis of the consequences of incidental take and rigorously evaluate management practices that are hypothesized to be the "best" to mitigate them.

Is there a best way to mitigate impacts of harvesting hay on productivity of grassland bird species? How solid is the evidence that inadvertent destruction of bird nests by timber harvesting significantly reduces the productivity of species affected? Is there solid evidence that the same harvest operations conducted outside the breeding season would have lesser midto long-term impacts on the same species? And how do the effects of other avenues of incidental take fishing bycatch, wind turbines, lit skyscrapers, and domestic cats - measure up to farming and forestry? The assessments undertaken by Environment Canada might have provided some insights; hopefully, the results will be published one day.

History has shown that conservation incentives weighted to clubs over carrots have not prevented the present state of affairs with respect to the conservation status of many species. The key question does not even pertain to clubs versus carrots; we need a mix of these two approaches. If governments really want to encourage voluntary actions from stakeholders that are designed to benefit conservation, there are only two important questions. First, will governments provide the necessary resources, as incentives to stakeholders, to participate in active adaptive management programs that have the potential to resolve key scientific uncertainties that underpin policy decisions? Second, will governments have the political courage, in the face of strong lobbying by some stakeholders, to permit scientific research in the form of active adaptive management that could reduce ecological uncertainty and its attendant costs? Only then will we be better positioned to learn which "best" management practices really are most efficient for lessening risks to bird species that are posed by incidental take or other potential threats.
En dépit de la sensibilisation grandissante de la population envers les impacts des humains sur les écosystèmes, de nombreux organismes sont tués accidentellement par des activités qui peuvent potentiellement affecter les taux vitaux des populations. En ce qui a trait aux passereaux, la destruction des nids par les opérations forestières ou agricoles durant l'été est un exemple de telles activités. Tout comme les prises accidentelles associées à la pêche commerciale, qui peuvent altérer significativement les réseaux trophiques des océans (Myers et Worm 2005), les "prises accessoires" d'oiseaux reliées à la destruction de nids ou à d'autres phénomènes comme les collisions avec des structures verticales ou la prédation par les animaux domestiques pourraient avoir des effets cumulatifs significatifs sur certaines espèces ou écosystèmes. Bien que les effets apparents de telles activités frappent davantage l'imagination du public que la destruction de l'habitat ou l'utilisation de pesticides en dehors de la saison de nidification, des analyses scientifiques sont tout de même requises afin de déterminer si, pourquoi et dans quelle mesure leurs effets sont dommageables.

Environnement Canada (EC) a entrepris ce type d'analyses suite à une enquête de la Commission de coopération environnementale (créée par l'Accord Nord-Américain de libre-échange)menée en raison de l'incapacité apparente du gouvernement canadien de faire appliquer la Convention concernant les oiseaux migrateurs (CCOM). Selon la CCOM, il est interdit de capturer ou tuer des oiseaux migrateurs ou encore de détruire leurs nids ou leurs œufs, et ce, quelle que soit la manière et en tout temps. Toutefois, en octobre 2010, une lettre provenant de la haute fonction publique de la Direction générale de l'intendance environnementale d'EC informait « toutes les parties intéressées » qu' après trois ans de recherche scientifique sur la myriade de façons dont les oiseaux et leurs nids peuvent faire l'objet de prise accessoire, EC "ne sollicitera pas d'amendements réglementaires liés à la prise accessoire; le ministère va plutôt se concentrer sur le développement de pratiques aidant de façon concrète à se conformer aux interdictions existantes. De plus, nous continuerons de travailler avec les parties intéressées pour créer un répertoire de meilleures pratiques qui aideront à la conservation des populations d'oiseaux migrateurs au Canada et qui procureront une base solide à ceux qui souhaiteront s'en servir."

À partir de cette information, il serait préjudiciable de conclure quoi que ce soit quant au destin de la recherche scientifique effectuée par le personnel 
d'EC au sujet des prises accessoires. Peut-être a-ton jugé qu'il serait trop onéreux de tenter de réglementer la prise accessoire sous toutes ses formes étant donné les ressources disponibles au gouvernement. De plus, il y avait peut-être des enjeux bi-nationaux, comme c'est souvent le cas lorsqu'il s'agit de la Convention concernant les oiseaux migrateurs. Si EC avait fait preuve de plus de transparence, les intervenants n'auraient pas à spéculer sur les motifs de cette décision. Néanmoins, il semble qu'une approche fondée sur la législation ne sera pas poursuivie dans le dossier des prises accessoires. Au contraire, EC sollicitera des propositions quant aux meilleures pratiques d'aménagement des parties intéressées, et ce, sur une base volontaire.

Un autre aspect de la décision qui ne ressort pas clairement est l'intention véritable d'EC. S'agit-il d'une transition de la méthode du "bâton" vers celle de la "carotte" (le point de vue d'un optimiste), i.e. utiliser des incitatifs pour promouvoir la conservation? Il est également difficile de déterminer le rôle que jouera la recherche scientifique dans l'évaluation de l'efficacité de cette initiative de conservation. Même les lois comme la CCOM, qui reposent sur des formulations très englobantes (Adkins Giese 2010) pour protéger les oiseaux migrateurs autorisent les gouvernements à permettre des prises accessoires dans la mesure où les actions dont il est question sont compatibles avec les termes de la loi et conformes aux objectifs de la CCOM, par exemple à des fins scientifiques et sous réserve d'approbation par les autorités compétentes. De la même façon, la législation sur les espèces menacées du Canada et au moins une de ses provinces, l'Ontario, permet certaines activités dans la mesure où leurs effets prévisibles à long terme seront probablement bénéfiques pour les espèces en question. Nous espérons que la décision de ne pas demander d'amendements aux règlements de la CCOM indique au moins l'intention du gouvernement de permettre des exceptions susceptibles d'encourager l' analyse scientifique des conséquences des prises accessoires et d'évaluer rigoureusement les pratiques d'aménagement présumées les «meilleures » pour en atténuer les effets.

Existe-t-il une meilleure façon d'atténuer les impacts de la récolte de foin sur la productivité des oiseaux des prairies? Jusqu'à quel point la destruction accidentelle de nids d'oiseaux par la coupe forestière a des effets significatifs sur la productivité des espèces affectées? Peut-on prétendre que les mêmes traitements sylvicoles effectués en dehors de la saison de reproduction auraient des conséquences moins grandes à moyen ou à long terme pour les mêmes espèces? Dans quelle mesure les autres phénomènes qui entraînent des prises accessoires - les prises accidentelles dans les filets de pêche, les collisions sur des éoliennes, des gratte-ciel illuminés ou la prédation par les chats domestiques - se comparent aux effets de l'agriculture et de la foresterie? Les évaluations entreprises par Environnement Canada auraient pu fournir des réponses; espérons que leurs résultats seront publiés un jour.

L'histoire a montré que les mesures incitatives pour la conservation fondées sur le bâton plutôt que sur la carotte n'ont pas empêché d'en arriver à l'état actuel en ce qui a trait au statut de conservation de plusieurs espèces. La question clé ne porte pas sur les mérites relatifs du bâton et de la carotte; nous avons besoin d'une combinaison de ces deux approches. Si les gouvernements tiennent vraiment à encourager des gestes volontaires des intervenants ayant pour objectif la conservation, il n' y a que deux questions vraiment importantes. Premièrement, estce que les gouvernements fourniront les ressources, sous forme d'incitatifs aux intervenants, qui leur permettront de participer à des programmes actifs de gestion évolutive ayant le potentiel de résoudre les incertitudes scientifiques clé qui sous-tendent les décisions en matière de politiques? Deuxièmement, est-ce que les gouvernements auront le courage politique, face aux puissants lobbys de certains intervenants, de permettre la recherche scientifique inhérente au concept de gestion évolutive qui pourrait réduire l'incertitude écologique et les coûts qui y sont rattachés? Ce n'est qu'à ce moment-là que nous serons en mesure d'apprendre lesquelles des "meilleures" pratiques de gestion sont effectivement les plus efficaces pour réduire les risques pour les oiseaux que présentent les prises accessoires ou les autres menaces potentielles.

Responses to this article can be read online at: http://www.ace-eco.org/vol5/iss2/art14/responses/ 


\section{LITERATURE CITED}

Adkins Giese, C. L. 2010. Spreading its wings: using the Migratory Bird Treaty Act to protect habitat. William Mitchell Law Review 36:11571178.

Myers, R. A., and B. Worm. 2005. Extinction, survival or recovery of large predatory fishes. Philosophical Transactions of the Royal Society BBiological Sciences 360:13-20. 